\title{
Kartierung der Vegetation der Schweiz nach einem Kilometer-Raster
}

Im Anschluss an die dringlichen Massnahmen des Bundes auf dem Gebiete der Raumplanung, vom 17. März 1972, zeigte sich das Bedurfnis, eine das ganze Land umfassende, einheitliche Uebersicht über den Wert einzelner Landschaften unter den Gesichtspunkten des Naturschutzes zu erstellen. Mit dieser Aufgabe betrauten die Abteilung $\mathrm{Na}-$ tur- und Heimatschutz des eidgenössischen Oberforstinspektorates und der Delegierte für Raumplanung die Pflanzengeographische Kommission der Schweizerischen Naturforschenden Gesellschaft.

Wegen der verhältnismässig geringen Mittel und der sehr knappen Zeit, mit der für die Ausführung einer solchen Uebersicht gerechnet werden konnte, war es notwendig, die anzuwendende Methode sowohl mit Bezug auf die pro Flächeneinheit einzusetzende Zeit als auch auf die mögliche Differenzierung der Resultate so effizient als möglich zu gestalten. Es war deshalb naheliegend, den Einsatz der elektronischen Datenverarbeitung von Anfang an als zentrale Methode einzuplanen.

\section{Methode der Datenbeschaffung}

Aufnahme und Auswertung erfolgen nach der Gitternetzmethode. Als Grundeinheit werden jene Quadrate verwendet, die auf allen Landeskarten der Schweiz durch die eingedruckten Koordinatenlinien definiert sind und die je $1 \mathrm{~km}^{2}$ messen. Für jeden einzelnen der über 40'000 Quadratkilometer der Schweiz werden eine Anzahl Daten erhoben und computergerecht notiert. Dabei leisten die verwendeten OMR-Karten (Optical Mark Reader) ganz ausgezeichnete Dienste. Durch eīnfaches Markieren eines Feldes mit schwarzem Bleistift sind die Angaben für die Uebertragung auf Magnetband brauchbar.

In Fig. 1 ist die für die Notierung der vorhandenen Vegetationstypen verwendete Karte abgebildet. Die erste Kolonne dient der Identifizierung der Karte selber, die nächsten 6 der Angabe der Koordinaten. Dann folgen 16 Kolonnenpaare, von denen je eines für die Eingabe einer Pflanzengesellschaft benötigt wird. Jeweils in der ersten der beiden Kolonnen wird die Nummer der Gesellschaft angegeben. Diese ist ein-bis dreistellig und darf nur Zahlen in aufsteigender Reihenfolge enthalten, Z.B. 234; die Nummer 432 wäre dagegen verboten, da sie in dieser Schreibart von 234 , 243 usw. nicht unterscheidbar ist. In der zweiten Kolonne folgen die Angaben uiber die Art des Vorkommens :
- Art der Feststellung ( $L=L$ iteraturangabe, $T=$ Feldbeobachtung, S=sicherer Analogieschluss, ?= fraglicher Analogieschluss. Diese Angaben sollen im Zweifelsfall zur Wertung der Sicherheit eines Vorkommens dienen).

- Ausdehnung des Vegetationstyps im Quadratkilometer in $4 \mathrm{klassen}$. ( $f=$ Fragment, weniger als 5 a; p=kleinflächig, 5 a bis 1 ha; durchschnittlich= 1 bis 50 ha, braucht nicht notiert zu werden; $\mathrm{g}=$ grossflächig, 50 bis $100 \mathrm{ha} / \mathrm{km}^{2}$ ).

- Ausbildung des Bestandes in 4 Klassen (-=duirftig; durchschnittlich braucht nicht notiert zu werden; b=gut; e=ausserordentlich gut ausgebildetes Vorkommen).

Vorkommen von in Ausdehnung und Ausbildung durchschnittlichen Beständen werden also nur mit der Art der Feststellung angegeben. Bei der Auswertung zeigte sich, dass diese Vereinfachung für die Datenbeschaffung eine sichere Kontrolle durch eine formelle Plausibilitätsrechnung stark erschwert.

Eine andere, nicht abgebildete Karte dient der Eingabe der häufigsten 33 Pflanzengesellschaften. Hier braucht die Nummer nicht verschlüsselt zu werden, die Angabe der Art der Feststellung genügt zur Notierung der betreffenden Gesellschaft.

Ein dritter Kartentyp ist in Fig.2 dargestellt; er dient zur Eingabe von verschiedenen Daten menschlicher Einrichtungen und der allgemeinen Oekologie:

- Einzelbauten ausserhalb von Siedlungen, die in der landesplanerischen Datenbank des ORL-Institutes der ETH nicht enthalten sind.

- Erholungseinrichtungen wie Strandbäder, Campingplätze, Sportplätze, Badanstalten und Parkplätze ausserhalb der Siedlungen.

- Landschaftsschäden wie Steinbrüche, Kies- und Lehmgruben, Schuttdeponien.

- Waldränder, unterschieden nach scharfer Abgrenzung und nach diffusen, ökologisch besonders wertvollen Rändern, mit Angabe der Länge im $\mathrm{km}^{2}$ in $3 \mathrm{Klassen}$ (weniger als $0.5 \mathrm{~km}, 0.5$ bis $2 \mathrm{~km}$, über $\left.2 \mathrm{~km} / \mathrm{km}^{2}\right)$.

- Hecken, Alleen und Windschutzstreifen nach den gleichen 3 Längenklassen wie die Waldränder.

- Ufer von Seen, Flüssen und Bächen, unterschieden nach künstlich verbauten und nach weitgehend naturnah ausgebildeten, ebenfalls in den drei Längenklassen der Waldränder.

- Geologische Unterlage in nach ihrer ökologi-

Or.0tto Hegg, Systematisch-Geobot. Institut der Universität Bern, Altenbergrain 21, 3013 Bern. 


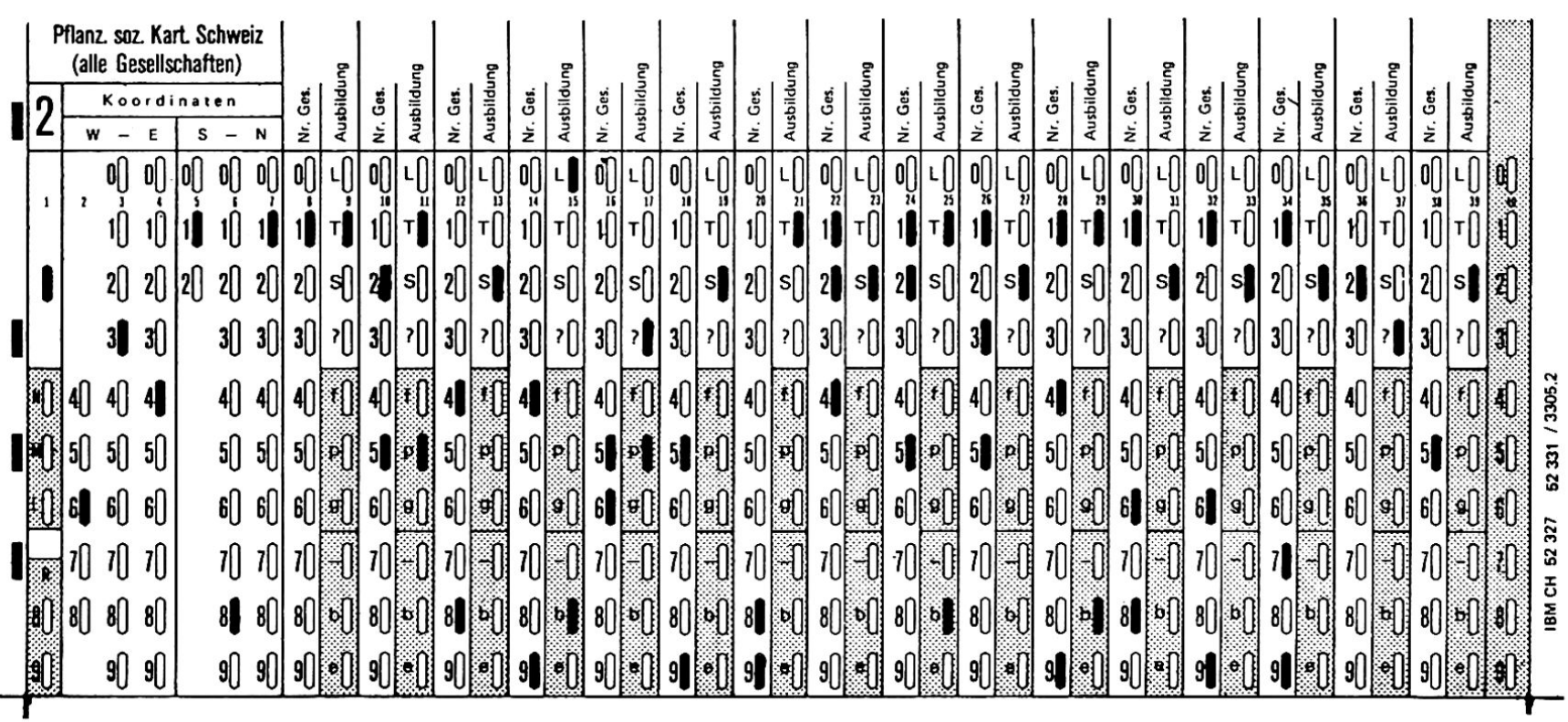

Fig.1: OMR-Karte zur Eingabe von vegetationskundlichen Daten (Details siehe Text).

schen Bedeutung zusammengefassten Klassen (Kalk, Mischgestein, Silikat; hart, weich; grobes und feines Lockermaterial; Sondergesteine). -Mittlere Meereshöhe im $\mathrm{km}^{2}$.

Modell der Pflanzengesellschaften der Schweiz

Eine wichtige Voraussetzung für die Durchführung der Kartierung ist die Annahme, dass die Vegetation der Schweiz heute, nach etwa 70 Jahren intensiver vegetationskundlicher Arbeit nach den Methoden verschiedener Schulen im Ganzen recht gut bekannt sei, gut genug, um ein Modell für das Vorkommen der Pflanzengesellschaften in der Schweiz aufzustellen. Dieses Modell'muss einerseits genügend differenziert sein, um für Naturschutzzwecke brauchbare Aussagen zu erlauben, es muss anderseits aber auch möglichst eindeutig sein in dem Sinn, dass es gestattet, für jede Stelle der Schweiz eine kurze Liste der dort oko- logisch möglichen Gesellschaften aufzustellen. Aus dieser kurzen Liste muss anschliessend mit $\mathrm{Hilfe}$ der neuesten Luftbilder der eine effektiv vorhandene Vegetationstyp zu ermitteln sein.

Die erste Arbeit an diesem Modell war, eine Liste der zu unterscheidenden Pflanzengesellschaften aufzustellen. Diese gesamtschweizerisch einheitliche Liste enthält 95 Typen, die etwa den Verbänden im pflanzensoziologischen System von Braun-Blanquet (1964) entsprechen. Es sind 33 Waldgesellschaften ( 18 Laub- und 15 Nadelwälder), 10 Strauchgesellschaften und 52 gehölzfreie Typen. Unter diesen sind 18 Trocken- und 18 Nassstandorte enthalten. Dazu kommen 22 weitere Einheiten, die durch starke menschliche Eingriffe bedingt sind, z.B. Fichtenforste,landwirtschaftliche Intensivkulturen, grossflächiger Gemuisebau usw., daneben aber auch solche mit recht hoher Bedeutung für den Naturschutz, so etwa traditionelle obstgärten, Wytweide oder Niederwald.

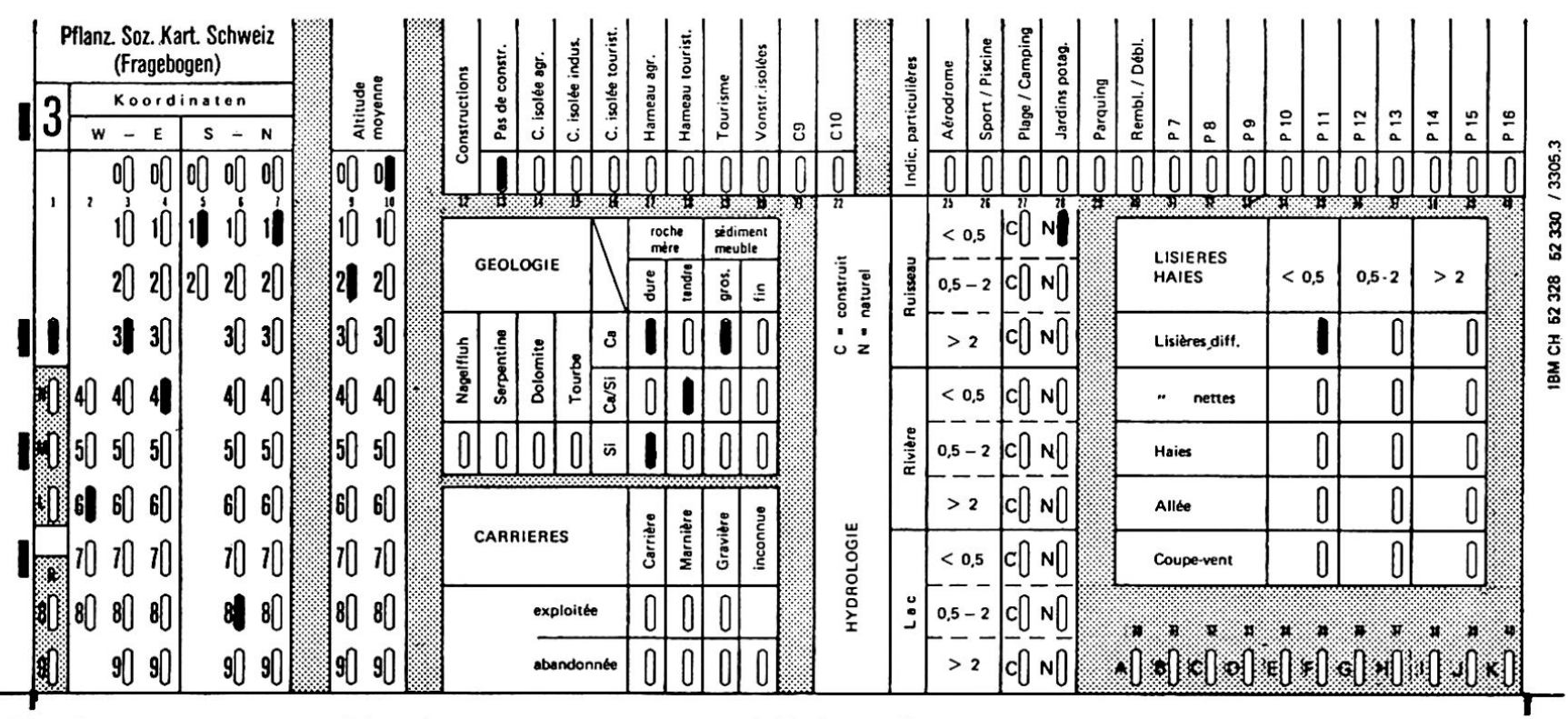

Fiy.2: OMR-Karte zur Eingabe von Daten zu menschlichen Einrichtungen und zur allgemeinen oekologie 
Der zweite Arbeitsgang war die Definition all dieser Vegetationstypen durch ein 0ekogramm ( $\mathrm{vgl}$. Béguin, Hegg und Zoller 1976 u. 1977). Es enthält Angaben über Meereshöhe, Relief, geologische Unterlage, Lokalklima, Boden, Wasserhaushalt und Vorkommen in den Höhenstufen der Schweiz. Es folgt eine ausfürliche floristisch-pflanzensoziologische Beschreibung mit Diskussion des $\mathrm{Na}-$ turschutzwertes.

Die Durchführung der nötigen Analogieschlüsse von den gut bekannten Teilen der Schweiz auf jeden einzelnen Quadratkilometer erfolgt im wesentlichen am Schreibtisch, mit Hilfe von topographischer Karte 1:25'000, geologischen Detailkarten (1:25'000 oder 1:50'000; z.T. mit der allerdings sehr detailreichen geologischen Generalkarte der Schweiz 1:200'000) und den neuesten Luftaufnahmen der schweizerischen Landestopographie, die ebenfalls ungefähr im Massstab 1:25'000 verfügbar sind.

Wenn trotz genauer Analysen dieser Unterlagen ein sicherer Entscheid nicht möglich ist, sind Kontrollen im Feld am Platz.

\section{Methoden der Datenverarbeitung}

Das auf Magnetband gespeicherte Datenmaterial gestattet eine vielseitige Auswertung mit EDV. Am häufigsten duirften irgendwelche Verbreitungskarten erwünscht sein, es sind aber auch verschiedene statistische Verarbeitungen vorgesehen.

Verbreitungskarten einzelner Vegetationstypen zeigen für jeden $\mathrm{km}^{2}$ mit einem einzelnen Zeichen an, wo ein bestimmter Vegetationstyp vorhanden ist. Dabei ist die Unterscheidung von $6 \mathrm{Klassen}$ möglich (z.B. nach Ausdehnung und Ausbildung). Ein anderer Typ von Verbreitungskarten zeigt die gesamte Information für jedes festgehaltene Vorkommen. Das erfordert unterschiedliche Zeichen für die Art der Feststellung $(4)$, die Fläche (4) und die Ausbildung (4) im $\mathrm{km}^{2}$ und ergibt so insgesamt 64 Möglichkeiten. Diese auf dem Schnelldrucker hergestellten Karten sind rel. schlecht lesbar und nur für Spezialzwecke sinnvoll. Ein Ausschnitt aus einer solchen Karte wurde publiziert von Kessler (1976).

Es ist auch leicht möglich, Karten herzustellen, welche zeigen, wieviele Gesellschaften aus einer bestimmten Gruppe von Vegetationstypen vorhanden sind. Die Gruppierungen können aufgrund pflanzensoziologischer Verwandtschaft oder nach Formationen, Standortsgruppen oder häufiger räumlicher Nachbarschaft zusammengefasst werden. Hier wird die Anzahl pro $\mathrm{km}^{2}$ ebenfalls in $6 \mathrm{Klassen}$ eingeteilt. Ein Beispiel einer solchen Karte ist in Fig. 3 wiedergegeben.

Selbstverständlich ist es auch möglich,eine Karte zu drucken, auf der die Gesamtsumme der Vegetationstypen pro $\mathrm{km}^{2}$ dargestellt ist.

Die zentrale Aufgabe solcher Karten besteht darin, eine Uebersicht über die ganze Schweiz zu geben und die Bedeutung der verschiedenen Landschaften für den Naturschutz zu veranschaulichen. Unter der etwas vereinfachenden Annahme, dass die
Gesellschaften der höheren Pflanzen eine gute Basis für die Ermittlung des gesamten Wertes einer Landschaft für den Naturschutz seien, dass also die Phytozönosen einen Massstab für den Reichtum und den Wert der Biozönosen abgeben, sind mehrere Möglichkeiten zur Abschätzung des Reichtums gegeben.

Vor allem eine kombinierte Berücksichtigung von Anzahl, Grösse und Bedeutung der vorhandenen Gesellschaften ergibt eine brauchbare Unterlage für den Naturschutz. Aus solchen Karten kann die Schutzwürdigkeit einer Landschaft mit genügender Zuverlässigkeit abgelesen werden.

Für ihre Herstellung gilt es also die verschiedenen Pflanzengesellschaften zu werten. Wir haben das unter Berücksichtigung der folgenden Gesichtspunkte gemacht, wobei deren Gewichtung unterschiedlich erfolgt:

Biologische Aspekte des Naturschutzwertes einer Pflanzengesellschaft:

- Seltenheit der Gesellschaft auf Grund der Anzahl Vorkommen

-Reichtum der Gesellschaft an seltenen Pflanzen und Tieren

-Bedeutung der Gesellschaft für diese Pflanzen und Tiere

-Empfindlichkeit der Bestände gegen menschliche Massnahmen (Düngung, Brachlegung usw.)

-Möglichkeit der Wiederherstellung eines entsprechenden Bestandes nach einer Zerstörung (irreplacability nach Tjallingij 1973; für die meisten Gesellschaften sehr schwierig und problematisch)

Menschliche Aspekte des Naturschutzwertes einer Pflanzengesellschaft:

-Bedeutung der Gesellschaft für die Landschaft als Umwelt des Menschen

-Bedeutung der Gesellschaft für die 0ekologie und Stabilität der Landschaft

-naturwissenschaftlicher Wert der Gesellschaft -ethische Gesichtpunkte

Unter Berücksichtigung dieser Gesichtspunkte ordneten wir jeder Pflanzengesellschaft einen Naturschutzwert zwischen 0 und $10 \mathrm{zu}$, wobei es möglich ist, Unterschiede zwischen den Grosstandschaften der Schweiz zu berücksichtigen, indem der gleichen Gesellschaft in 13 Regionen verschiedene Werte gegeben werden können.

Der Computer berechnet nun auf Grund der im $\mathrm{km}^{2}$ vorhandenen Pflanzengesellschaften unter Berücksichtigung der Fläche, der Ausbildung und der verschiedenen Naturschutzwerte der Gesellschaften einen gesamten Naturschutzwert, der, unterteilt in 6 Klassen, in einer Karte dargestellt werden kann.

Besonders wichtig für die praktische Anwendung dürten Karten sein, die den Naturschutzwert für ökologisch einigermassen einheitliche Gruppen von Vegetationstypen darstellen, also etwa für die Nassvegetation, Trockenvegetation, Waldvegetation usw.. Die Auswirkungen von menschlichen Eingriffen bleiben innerhalb von solchen Gruppen vergleichbar, während bei Berücksichtigung eines Gesamtwertes für die komplette Vegetation unter Umständen der gleiche Eingriff einzelne Pflanzn- 
gesellschaften fördern könnte, während andere gänzlich vernichtet werden. Es muss deshalb auch zu jeder einzelnen Gesellschaft ebenso wie zu den verschiedenen Synthesekarten im Text auf die Auswirkungen häufiger menschlicher Eingriffe eingegangen werden, damit mögliche Folgen abgeschätzt werden können.

\section{Literatur}

Béguin,C., Hegg,0., Zoller,H. (1975):Pflanzensoziologisch - ökologische Kartierung der Schweiz mit der Gitternetzmethode zu Naturschutzzwekken. Internat. Ges. f. Vegetationskunde, Symposium 1974 in Rinteln, im Druck.

-.-.-(1975): Landschaftsökologisch-vegetationskundliche Bestandesaufnahme der Schweiz zu Naturschutzzwecken. Verh. Ges. f. Oekologie, Er langen 1974, S. 245-251.

-.-.-(1976): Utilisation d'écogrammes pour une étude éco-phytosociologique de la Suisse. Doc. Phytosociol. Fasc. 19-20, S. 89-97.

-.-.-(1977): Ecograms of Swiss forest associations. Natural. Canadien 104, S. 5-9.

Braun-Blanquet J.(1964): Pflanzensoziologie. Springer, Wien, 3.Ed. 865 S..
Kessler,E.(1976): Grundlagen für die Ausscheidung von Schutzgebieten in der Schweiz. Natur und Landschaft 51, Nr. 5, S. 143-149.

Tjallingij,S.P.(1973): Unity and Diversity in Landscape. Contrib. 3rd intern. symposium Content and Object of the Complex Landscape Research. Bratislava.

Fig.3: Karte der Anzahl von ungedüngten Rasengesellschaften im Quadratkilometer.

Folgende Gesellschaften sind berücksichtigt: Xerobromion, Mesobromion, Seslerio-Bromion, Andropogonetum grylli, Festucetum spadiceae, StipoPoion carniolicae, Stipo-Poion xerophilae, DraboSeslerion, Oxytropo-Elynion, Seslerion, Caricion ferrugineae, Molinion, Caricion canescenti-fuscae, Caricion davallianae, Caricion curvulae, Festucetum variae, Laserpitio-Poion violaceae, Caricion sempervirentis, Nardion, Calluno-Genistion, Calamagrostion, total 20 Gesellschaften. Es handelt sich durchwegs um Vegetationstypen, die für den Naturschutz wertvoll sind und in denen viele seltene Pflanzen und Tiere ihre Hauptverbreitung haben.

Legende: I: 1 Gesellschaft im Quadratkilometer vorhanden; 2: 2 Ges.; 3: 3-4 Ges.; 4: 5-6 Ges.; 5: 7-8 Ges.; 6: über 9 Ges. (maximal 11); 7: Seefläche; 8: Landesgrenze.

\section{Literaturbesprechung}

MANSHARD Walter, Die Städte des tropischen Afrika - Band 1 von Urbanisierung der Erde, herausgegeben von W. Tietze, Bornträger Berl in u. Stuttgart 1977, 258 S., 104 Abb.,33 Tab.,DM98.--

Verstädterung war lange als eine Erscheinung der Industrieländer gesehen worden. Erst in den letzten Jahren setzte sich die Erkenntnis durch, dass sich in den Ländern der Dritten Welt ein Vorgang der Verstädterung abspielt,der eigenen Charakter hat und seine eigenen Probleme birgt.Das vorliegende Werk liefert zu diesem immer wichtiger werdenden Fragenkreis einen beachtenswerten und wertvollen Beitrag. Abgesehen von einer kurzen aber lesenswerten - Einführung und einer etwa einen Fünftel des Umfanges ausmachenden Zusammenfassung bietet es eine sorgfältig dokumentierte, nach Regionen geordnete Sammlung von Fallstudien mit eingeschalteten Ueberlegungen genereller Natur. Manshard verzichtet auf alarmierende Prognosen - wie sie kürzlich in der Presse breiten Raum fanden - und stellt die Dinge dar,wie sie sind. Er weist auch darauf hin, dass sich zwar die Verstädterung im tropischen Afrika nur schwer mit den theoretischen Modellen,wie sie vor allem in den USA entwickelt worden sind, begreifen lässt,verzichtet aber andererseits auch darauf, neue Modelle zu entwickeln und allgemein gilltige Theorien zu postulieren. Der Titel ist darum im Sinne einer regionalen Begrenzung und weniger einer systematisch-theoretischen Ausrichtung zu verstehen. Der Verfasser,der Afrika aus langjähriger eigener Anschauung kennt,muss sich verständlicherweise gleichwohl über weite Strecken auf die Arbeiten anderer stützen. Dadurch kommt eine gewissevielseitigkeit, aber auch Unausgeglichenheit in die Darstellung. Zu denken ist etwa daran,dass im einen Falle die Stadt lediglich für sich,im anderen aber auch in ihren Beziehungen zum Umlande gewürdigt wird. Für den Geographen - ausgenommen ausgesprochene Stadtspezialisten - ist die Stadt in erster Linie ein Teil eines grösseren Ganzen und Arbeiten, die dies nicht einbeziehen, haben vornehmlich elementaranalytischen Wert.

Hans Boesch 